Research Article

\title{
The Effects of Implementing Clinical Supervision Model on Supervisors' Teaching Perspectives and Qualifications: A Case Study in an EFL Context
}

\author{
Ehsan Khaef $(i D)$ and Amin Karimnia \\ Department of English Language, Islamic Azad University, Fasa, Iran \\ Correspondence should be addressed to Amin Karimnia; aminkarimnia@yahoo.coom
}

Received 7 October 2021; Accepted 23 October 2021; Published 15 November 2021

Academic Editor: Ehsan Namaziandost

Copyright (c) 2021 Ehsan Khaef and Amin Karimnia. This is an open access article distributed under the Creative Commons Attribution License, which permits unrestricted use, distribution, and reproduction in any medium, provided the original work is properly cited.

\begin{abstract}
This study set out to determine the possible impacts of implementing the Clinical Supervision Model (CSM) on supervisors' philosophy of teaching, in both theory and practice. In order to gather the required data, two highly experienced supervisors were recruited according to the degree of homogeneity of the educational context in which they were working. By applying qualitative case study design, the required data were collected by conducting two distinct phases of classroom observations, accompanied by semistructured interviews. Considering the nature of the investigation, it was decided to employ the Transcendental Phenomenology $(\mathrm{TPh})$ approach to analyze the data in order to determine the supervisors' accurate perceptions and lived experiences regarding the nature of taking on a supervisory role and the effects of implementing CSM on their teaching career. The empirical findings in this investigation provided a new understanding of the probable effects of implementing CSM on supervisors' general attitudes towards both teaching and supervisory careers. The results of the study indicated that CSM can be potentially effective in encouraging critical thinking, providing more constructive feedback in postobservation meetings, and improving the teaching quality of both teachers and supervisors.
\end{abstract}

\section{Introduction}

One of the prime concerns of any educational system is to create an effective learning environment that improves students' academic skills and competencies as well as their social and emotional growth. In the light of the novel approaches to teaching methods and strategies in the new millennium, systematic classroom observation emerged as an important multipurpose vehicle for educational policymakers to appraise the instructors' performance in order to enhance teaching quality and improve students' outcomes.

The traditional supervision model was conducted by single individuals (supervisors) with the purpose of faultfinding in an authoritative, threatening, noncollaborative manner. It exclusively focused on teachers, not on the teaching process or students' achievements. Due to the variety of problems caused by traditional supervision, the cooperation between teachers and supervisors was minimal [1]. In the view of O'Leary [2], the English education system in recent times has been under an ongoing reform agenda. In accordance with a new policy focus, a package of reforms and initiatives targeted at evaluating achievement and raising standards with the stated goal of enhancing the quality of teaching and learning was developed. As a result, classroom observation was identified as a key tool in the improvement of teaching practice and considered to be the principal means of reinstating what is regarded as "core" in education ([2], p.17).

Nel and Fouche [3] identified supervision as one of the contributing factors which predispose teachers to gradual educational development. They believe that, in recent years, effective supervision practices have been developed for the purpose of securing teachers' emotional support, self-acceptance, personal growth, and autonomy. The most crucial 
point noted across these topics was the quality of the supervisory relationship. Many authors (Peake, Nussbaum, and Tindell [4]; Worthen and McNeill [5]) as cited in the work of Nel and Fouche [3] have acknowledged that the relationship between teachers and supervisors must consist of warmth, acceptance, respect, understanding, and trust. Experiencing these features may not only lead to participants' professional growth but also result in teachers' and supervisors' constructive collaboration.

Clinical supervision, according to Cogan [6], can be categorized into eight sequential steps:

Phase 1: establishing the teacher-supervisor relationship

Phase 2: planning with the teacher

Phase 3: planning the strategy of observation

Phase 4: observing instruction

Phase 5: analyzing the teaching-learning processes

Phase 6: planning the strategy of the conference

Phase 7: the conference

Phase 8: renewed planning

Goldhammer [7] devised a five-phase clinical supervision approach that entailed instructors and supervisors engaging in a reflective conversation.

Phase 1. Preobservation Conference. The purpose of this phase was to establish a conceptual framework for the observation. The teacher and supervisor determined the details of the observation during this phase.

Phase 2. Classroom Observation. Using the framework outlined in Phase 1, the supervisor observed the teacher in the classroom.

Phase 3. Analysis. The supervisor gathered the data from the observation with the goal of assisting teachers "in creating evaluations of their own teaching" (p. 63).

Phase 4. A Supervision Conference. During this phase, the teacher and supervisor discussed the data. The teacher was invited to reflect on and explain his or her teaching methods. This step could also include providing the teacher with “didactic aid" (p. 70).

Phase 5. Analysis The supervisor's "practice was scrutinized with all of the rigor and for essentially the same goals that teacher's professional behavior was assessed theretofore," according to the report (p. 71).

Clinical supervision, according to Acheson and Gall [1], is grounded in the belief that teachers will appreciate a supervisory style that is concentrated on their own interests and expectations. Clinical supervision's major goal is to help teachers enhance their professional performance and instructional effectiveness $[1,8]$.

The primary goal of implementing the Clinical Supervision Model (CSM) is to establish a collaborative relationship between supervisors and teachers in order to maintain a responsive educational environment that fosters students' holistic learning. Most of the previous studies in the field of CSM have mainly focused on the privileges of implementing CSM over the traditional model of supervision and the possible impacts of CSM on teachers' overall performance. As a consequence, the existing literature fails to determine the possible effects of implementing CSM on supervisors' educational philosophy and teaching techniques. To the best of the researchers' knowledge, there have been very few studies about the possible impacts of applying CSM on supervisor's philosophy of education, particularly in EFL contexts. The main purpose of this study is to gain a deep insight into the possible impacts of implementing CSM on supervisors' teaching perspectives in both theory and practice. This investigation aims to answer the following research questions:

(1) What are the potential impacts of taking a supervisory role on observers' critical thinking about pedagogical issues?

(2) What are the effects of implementing CSM on supervisors' educational philosophy and teaching style in practice?

1.1. Literature Review. In 1973, Cogan declared that supervisors should look for "critical incidents" that "substantially prevent desired learning” (p. 172). He also emphasized that the supervision process should be seen as an essential aspect of the continuous improvement process in teaching. Cogan went on to say that clinical supervision is a continuation of the teacher's professional training. This means that teachers are constantly committed to improving their teaching quality, as demanded by all professionals. In this sense, the teacher should not be treated as a person rescued from incompetence or supported in their pitfalls. In supervisory processes, teachers must be perceived as specialists who are further trained and broaden their skills ( $p$. 21). Cogan underscored the fact that a supervisor's personal teaching model could affect his/her ability to provide effective feedback to teachers.

According to Marzano et al [9], few advances in the realm of education have spread as rapidly as clinical supervision. Clinical supervisory models, which were developed in the late 1950s and documented in depth in publications released in the late 1960s and early 1970s, spread like wildfire. By 1980, almost $90 \%$ of school administrators employed some form of Clinical Supervision Model (Bruce and Hoehn, 1980), as cited in the work of Marzano et al. [9].

Wiedmer [11] believes that the clinical supervision method is a collaborative and supportive approach to the professional development of teachers which creates a meaningful bond with peers. Teachers feel free to set "stretch" goals (those for which there is no certainty) rather than "safe" ones in this environment. Clinical supervision provides an opportunity for collegial support, allowing for the exchange of ideas and reflection on professional practice, 
as well as offering encouragement while working towards educational objectives [11].

Bello and Olaer [12] stated that powerful teaching leads to effective learning. Teachers can be effective when they possess educational competence. A competent teacher is flexible, possesses the abilities and capabilities, and is able to effectively and efficiently utilize her/his potential as required by the job. They measured the influence of clinical supervision on teachers' competence, and it was revealed that clinical supervision significantly affects the pedagogical competence of teachers. They concluded that the more teachers are supervised, the better they will become.

In this regard, Rahmat et al. [13] believe supervision should be carried out in order to guarantee quality learning, which means that the success of supervision is measured by increasing student learning achievement. Supervisors play an important role in enhancing the quality of teachers to ensure a better quality of learning. Clinical supervision is influenced by behavioristic theory. The word "clinical" refers to the face-to-face interaction between the teacher and the supervisor in problem solving reflexes, directed at per class, and focused on the teacher as an agent of change. It allowed supervisors and teachers to discuss together and analyze learning problems that arose in the classroom and find solutions to those problems. Rahmat et al. [13] emphasized the fact that teachers need technical assistance, which is usually in the form of academic supervision conducted by the school supervisor. They believe that a supervisor needs to be able to fully understand the different steps involved in the implementation of clinical supervision, including planning, implementation, and evaluation.

Babaeer [16] found that supervisors' past experience with supervision as teacher trainees prior to serving and university supervision plays an important role in shaping their conceptions of supervision. The participants in his study stated that they regularly relied on design of supervision based on their past experiences as a teacher trainee and on the observation of their current colleagues, since they did not have formal professional training on the subject of supervision. They all identified the magnitude of growing a context that would assist in conducting their supervision and, subsequently, preservice teacher learning. The information in Babaeer's [16] study confirmed that constructing relationships between university supervisors, preservice teachers, and cooperating teachers are the key points in assisting the university supervisors to enact supervision. The findings of this study highlighted that the participants chose to intensify the concept of supervision as an enhancement of the learning, growth, and improvement of preschool teachers, rather than focusing too much on supervision as evaluation (Baecher and Burns, 2020).

Marashi and Bani-Ardalani [16] asserted that clinical supervision can help EFL teachers overcome the state of exhaustion and burnout. According to them, since both supervisors and teachers have a harmonious and collaborative relationship through a clinical supervision program, professional development takes place. The reason is that teachers feel they have a prominent role in the process of supervision. They concluded that teachers who collaborated in CSM showed a positive attitude towards supervisory practice in general.

McGhee and Stark [17] point out that faculty members should promote more effective practices that place the teacher at the center of the process and specifically involve teachers in the supervisory programs. To this end, they conducted prospective educational supervisions accompanied by developmental supervisory behavior and the clinical supervision cycle in combination with solution-focused language and strategies. Ultimately, it revealed that teachers' active role in the process of supervision led to increased confidence and more effective educational outcomes.

Bencherab and Al Maskari [18] declared that CSM is an ethical, impartial, stress-free process which is basically teacher-centered. Because of the dual relationship between teacher improvement and student performance, when used correctly, CSM serves a number of worthy purposes. In their words, there are several features that distinguish clinical supervision from other types of supervisory practices. In this regard, Hopkins and Moore [19] as cited in Bencherab and Al Maskari [18] have recognized the following traits as the most significant ones: emphasizing the strengths rather than the weaknesses of the teachers, focusing on teachers' educational growth, and establishing formative rather than summative evaluation.

The results of the literature study by Ibara [20] indicate that the collaborative clinical supervision approach can be successfully applied to most of educational contexts. As a supervisory technique, the collaborative approach includes a formative face-to-face interplay between a supervisor and a teacher and consequently gives a productive tool for supervision in teacher education. The use of the approach implies that the supervisor and the teacher actively participate in the decisions made and strive to develop a relationship throughout the supervision cycle in order to promote the long-term professional improvement of both teachers and supervisors.

\section{Methodology}

2.1. Research Design. This study utilized qualitative case study design to gain deep insights into the lived experiences of two well-reputed supervisors who have been observing EFL classes for a long time. In order to analyze the data, it was decided to apply the Transcendental Phenomenology $(\mathrm{TPh})$ approach in order to determine the supervisors' accurate perceptions and lived experiences over the nature of taking on a supervisory role and the effects of implementing the Clinical Supervision Model (CSM) on their professional career. According to Cohen et al. [21], phenomenology is a theoretical framework that seeks to promote studying direct experience at face value and sees behavior as being determined by experiential phenomena rather than external, objective, and physically specified reality. In the view of Creswell [22], a phenomenological investigation explores a notion, an idea, or a reality for a set of individuals indicating what all share in common while they are experiencing an event. Since the analysis of supervisors' experiences plays a pivotal role in this investigation, Cohen's definition is 
relevant to the objectives of the current study. On the other hand, because part of the study was seeking to find similarities between the participants' perceptions of the nature of supervision in general, Creswell's notion could also be applicable.

According to the definition provided by Moustakas [26], phenomenological research is a method of inquiry in which the researcher identifies the essence of participants' descriptions of a phenomenon. Moustakas [26] argues that phenomenology is both a philosophy and a method for understanding life experiences. The procedure entails examining a small number of subjects over a lengthy period of time in order to discover patterns and relationships of meaning. Laverty [27] believes that Transcendental Phenomenology enables the formation of a perspective that incorporates external, physical, and isolable stimuli, which results in capturing the "essence" of the phenomenon.

2.2. Context of the Study. The study was conducted at the Adults' Department of the Iran Language Institute (ILI), one of the oldest language institutes in Iran, where there is a systematic classroom observation for evaluating teachers' performance. The ILI classes are held twice a week, with each session lasting 90 minutes. The teaching methodology is basically the Grammar-Translation Method (GTM), which is conspicuously teacher-based. The ILI teachers, tightly controlled by the supervisors, need to be observed unannounced and without prior notice every semester. They are supposed to stick to the ILI's methodology and guidelines meticulously.

2.3. Participants. Owing to the nature of research design and methodology, it was decided to adopt nonprobability, purposeful/homogenous sampling to recruit the target participants. In this way, the researcher was able to choose a sample on the basis of the study's objectives and demographical knowledge. In order to recruit the proper participants, several emails, including a brief description of the current investigation, were sent to some highly experienced ILI supervisors who seemed to be potentially capable of meeting the theoretical framework and objectives of the study. Two supervisors were interested in participating in the project. Each participant is described briefly below (in terms of maintaining the participants' privacy and anonymity, their real names were replaced with pseudonyms).

2.3.1. Participant \#1: Nima. Nima is a sixty-year-old supervisor who has been working at the ILI for about thirty years. He holds a Bachelor's degree in English Literature and Master's degree in EFL. He loves teaching; it has been his dream job since he was a child. Nima is the ILI's reputable observer and teacher-trainer. He has been observing ILI classes for more than two decades and knows the highs and lows of supervision. Currently, he supervises different departments of the ILI (Children, Young Adults, and Adults).
2.3.2. Participant \#2: Shima. Shima is one of the ILI's experienced teachers/supervisors. She got her Bachelor's degree in English Translation and Master's degree in EFL. She has been teaching English at high schools in Shiraz (a large city in Iran) for about twenty years. Shima started teaching at the ILI when she was 22 . She has been observing ILI classes for about 13 years. Currently, she supervises in the ILI's Adults Department.

2.4. Data Collection Procedure and Instruments. The first instrument used in this study was a preinterview session with recruited participants so as to get familiar with their personal traits and general attitudes towards classroom observation. During the preinterview sessions, the researcher made a brief introduction and asked the participants to introduce themselves. Then, some general issues regarding classroom observation were discussed and the purpose of the study was clearly stated. Furthermore, the researcher discussed different aspects of CSM with the participants (in both theory and practice) in order to make them aware of the whole process and resolve the possible ambiguities. Finally, the researcher clearly explained the process of data collection method in order to gain the participants' full consent. The preinterview protocol is provided in Appendix A.

The second instrument was a two-part series of purposeful observations of the supervisors' classes in a specific session, both before and after the implementation of CSM. In the first phase, the researcher observed the supervisor's class as a teacher. Then, the same class was observed by the researcher and handled by another teacher where the supervisor was taking a supervisory role. The researcher attended the class full-time and carefully recorded what happened in the postobservation session (the supervisor's attitude, recommendations, etc.). At the end of the first phase, a semistructured interview was conducted with the supervisors. Then the supervisors were asked to implement the principles of CSM in the following observations. The same process was gone through in the second phase, this time, followed by semistructured interviews after each step. The abovementioned process is shown in Figures 1 and 2 below.

Having granted the written consent of the ILI, a time table was arranged to observe supervisors' classes. First, Nima's Intermediate 2 class, Session 6 was observed. According to the syllabus, Nima was supposed to check the Workbook, ask a couple of students the previous passage, and teach a dialog and some grammar points. The listening part should also be covered at the end of the session. The researcher closely observed the class and took detailed notes of what happened in the classroom.

A few days later, Nima was supposed to observe Babak (a pseudonym), a teacher who was teaching level Intermediate 2, for the first time (the same session, the same content: Session 6). The researcher observed Babak's class under the presence of Nima as a supervisor and got a copy of Nima's observation form about Babak's performance. The observation form consisted of Nima's detailed opinions about 


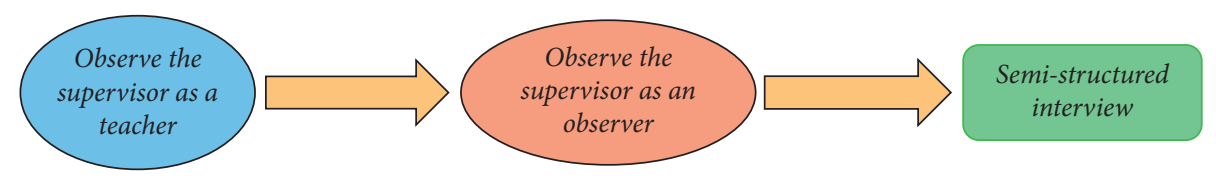

FIgURE 1: Phase 1 (before the implementation of CSM). Observation of supervisors' classes both as a teacher and as a supervisor.

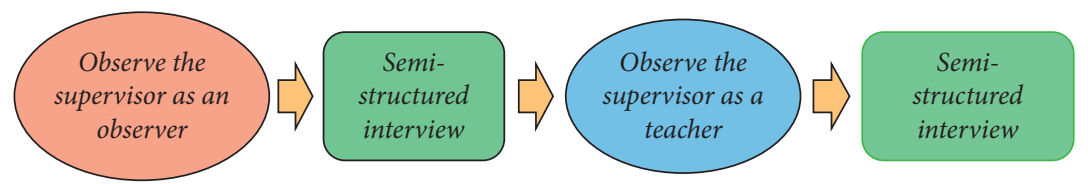

FIgURE 2: Phase 2 (after the implementation of CSM). Observation of supervisors' classes both as a teacher and as a supervisor.

Babak's performance and his overall recommendations for a better performance in the future.

On the next step, a semistructured interview was conducted with Nima to discuss his performance both as a teacher and as a supervisor. The same process went through for Shima. In the second phase of the classroom observations series, Nima and Shima were asked to implement the protocols of CSM in their supervisory careers. Following that, the same steps were taken. The researcher conducted interviews after each step. The interviews with the participants were recorded and transcribed. An enormous amount of information was also gathered during the observations. The questions of semistrutted interviews in the first phase and second phase of the study are provided in Appendix B and Appendix C, respectively.

2.5. Data Analysis Procedure. In order to analyze the data, Moustakas' [26] adaptation of Van Kaam's phenomenological data analysis was used, which consists of several steps:

(1) Horizonalization. Treat each piece of information equally. No quote or extract is more significant or valuable than the others. This is where you start the basic classification and contextualizing process by writing down every quote that is pertinent to the experience/phenomenon being investigated.

(2) Reduction and Elimination. This is where you take your quotation list and try to answer the following questions: (1) is there any harmonious relationship between this quote and the participants' lived experience of the phenomenon? (2) Can this quote be reduced to its underlying meaning? The quote will be removed if the answer is no to any of these questions. This can enormously help to distinguish the experience's invariant components from superfluous and extraneous data.

(3) Checking the Themes against the Data. When you have developed the themes, it is time to compare them to the dataset. This is to ensure that the themes accurately reflect the participants' experience and contribute to the participants' story.

(4) Creating Individual Textual Description. An individual textual description needs to be written for each participant in this stage. These are fragments and quotes from the participants which are used verbatim in the description.

(5) Creating Individual Structural Descriptions. This step necessitates the use of creative innovation. You create distinct structural descriptions for each participant. These are summaries that explore the emotional, social, and cultural ties that exist between what participants say. This is where the major interpretation is provided.

(6) Create Composite Textual Descriptions. In this step, you need to create a table outlining all of the themes from each participant. This will help recognize recurring and noticeable themes among all the participants, which is exactly what you require. This composite description will summarize what participants said during their interviews and highlight similar themes from the phenomenon's lived experience.

(7) Create Composite Structural Description. In this stage, you need to examine the emotional, social, and cultural links between all of the participants' experiences. This is where you discuss the components of their experiences that they have in common. Participants could have come from a low socioeconomic background or be of a specific race. It makes no difference which aspects are in common; what matters is the fact that they are all taken into account. This is when you start thinking about what factors most influence their experiences and what factors inform their experiences.

(8) Create Composite Structural-Textual Description. This step is also called "Synthesis." This is where you combine the textual and structural aspects of the phenomenon to develop a profound understanding of the phenomenon. This is how the phenomenon's lived experience is summed up.

Moustakas [26] asserts that there are four integral events in the process of Transcendental Phenomenology:

(1) The Transition. A stage where everything is viewed freshly as though for the first time, moving beyond the ordinary experience to the hinterland of pure ego. 
(2) The Epoche. The researcher must leave aside prejudice, biases, judgement, and preconceptions about the phenomenon to analyze the contribution of research participants objectively (Husserl, 1977, as cited in the work of Moustakas [26]).

(3) Transcendental Phenomenological Reduction. It is the moment of withdrawal from the natural attitude and from the everyday world towards the intersubjective level of transcendental ego.

(4) Imaginative Variation. It allows the researcher to incorporate the "infinite multiplicities of actual and possible cognitions that relate to the object... and can thus somehow go together to make up the unity of an identifying synthesis of meanings and essences" (Husserl, 1997, page 63).

Figure 3 depicts various steps in implementing the phenomenological approach.

2.6. Findings and Results. This qualitative case study set out to determine the effects of implementing CSM on supervisors' teaching philosophy in both theory and practice. The investigation was conducted at the Iran Language Institute (ILI) in which the supervisors play a crucial role in the process of supervision. The required data was gathered from two experienced supervisors through a series of classroom observations followed by semistructured interviews. In order to analyze the data, Moustakas' [26] revision of Van Kaam's Transcendental Phenomenology was conducted. Six broad themes emerged from the analysis of the data, gathered from the two participants of the study. The findings are based on the participants' perceptions of implementing CSM based on their personal experiences. Because each participant had different experiences, first we decided to categorize the generated themes separately and then convey the essence of their experiences together. Table 1 provides the themes of each participant generated from the analysis of the data gathered from the interviews and observations.

2.6.1. Participant \#1: Nima. During the first interviews, Nima was rather skeptical about the efficiency of CSM. As time went on, he got more interested in the process. In the first interview, after observing Nima's classes both as a teacher and as a supervisor, he simply defined his supervisory role as someone who is experienced enough to help novice teachers improve their teaching skills. During the observation of Babak's class on the same unit, he was busy taking extensive notes. In the postobservation meeting with Babak, first, he mentioned some of his pronunciation mistakes. Then he gave him some general advice about time and classroom management. Finally, he asked him to "stick to the methodology" and go through the steps in chronological order. Babak uttered a few words during the postobservation meeting and just signed the observation form. The summative and judgmental nature of the supervision process was quite tangible.
After Nima's observation of Babak's class, when the researcher asked Nima whether his supervisory role had affected his own style of teaching, he said: "Well, sure it did." I asked him to elaborate on his answer. He continued: "Sometimes I observe highly knowledgeable teachers whom I deeply admire. They efficiently handle the classroom with a wide variety of sophisticated techniques which I sometimes implement in my own classes..." As an experienced supervisor, Nima seemed to be fully aware of the possible impacts of taking on a supervisory role on observers' reflective notions and teaching style. Nevertheless, his conventional approach to the classroom observation was questionable because he did not have a fruitful collaboration with the teacher during the supervision process.

In the second phase of the observation series, I asked him to implement the protocols of CSM. He arranged the time with the teacher to observe his class and informed him of the observation date and time. After the observation, he gave the observation form to the supervisee and asked him to take a look at the form and reflect on his performance. The postobservation meeting was held a few days later. "It was remarkably fruitful!," Nima declared. I asked him to explain more. "Well... I arranged the postobservation meeting with Babak a few days after the observation and discussed Babak's performance with him, allowed him to express his views about my opinions and recommendations. He had some clever ideas about presenting different parts of the unit that he had covered (especially the warm-up for conversation). He even made some interesting suggestions about presenting the follow-up section in the listening part. I may try it in my own classes..." "Did you happen to compare your own performance as a teacher with Babak?" I asked. He replied, "Yeah... I also discussed my own ideas with him, and we somehow came up with an agreement... I think by the implementation of CSM, we let the teachers BE HEARD. Actually, we used to stifle their voices.... I filled out a new observation form and included Babak's views. I also asked him to document his own views in the final draft of the observation form...."

2.6.2. Participant \#2: Shima. Shima was really interested in the process from the outset. During the first interview, she expressed her dissatisfaction about the ILI's current supervision process and affirmed that she was required to apply it this way. In her opinion, the ILI's observation system is not formative and developmental and has hardly ever had positive effects on teachers' performance. In the first phase of the supervision process, she observed Nahid, a teacher with six-year experience who was observed to get promoted to teach upper levels. In the postobservation meeting, she thanked the supervisee for her performance and appreciated her knowledge and expertise. Then, she mentioned some grammatical and pronunciation mistakes that Nahid had made and asked her to involve more students in the process of teaching grammar. Nahid was silent during the postobservation meeting. At the end, she just asked, "Where should I sign?!" It could be inferred that teachers' views were completely ignored in the process of supervision. When I shared my views about the judgmental nature of the current 


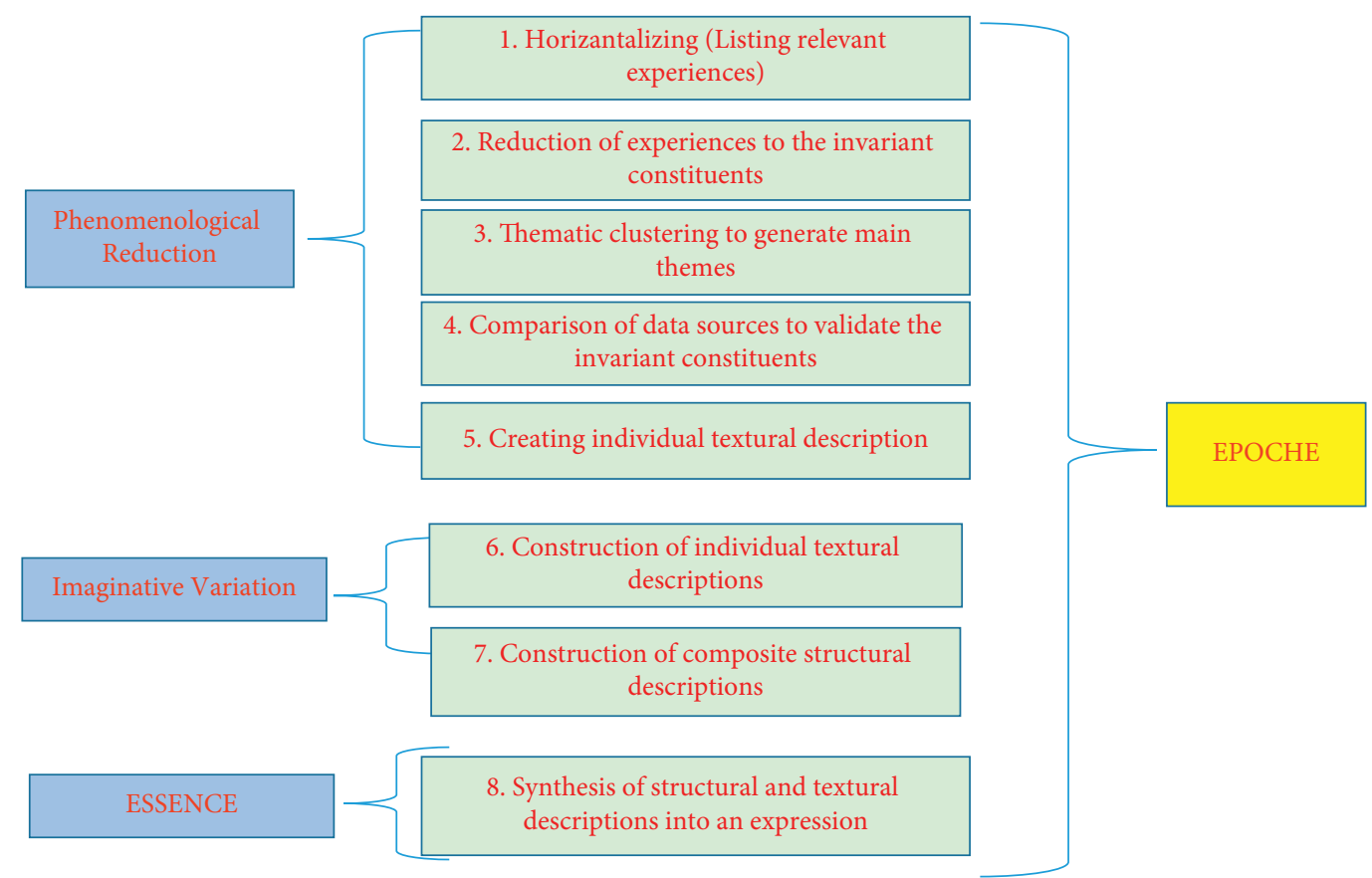

FIgURe 3: The steps of phenomenological data analysis by Moustakas [26].

TABLE 1: Themes for each participant.

\begin{tabular}{lccc}
\hline Participants & Theme 1 & Theme 2 & Theme 3 \\
\hline Nima & $\begin{array}{c}\text { CSM prompts reflective } \\
\text { notions about teaching }\end{array}$ & $\begin{array}{c}\text { CSM encourages collaborative interaction } \\
\text { with teachers }\end{array}$ & $\begin{array}{c}\text { CSM leads to more effective documentation } \\
\text { of the supervision process }\end{array}$ \\
\hline Shima & $\begin{array}{c}\text { CSM provides teachers with } \\
\text { more fruitful feedback }\end{array}$ & $\begin{array}{c}\text { CSM redefines the nature of the relationship } \\
\text { between teachers and supervisors }\end{array}$ & $\begin{array}{c}\text { CSM fosters developmental, formative } \\
\text { supervision }\end{array}$ \\
\hline
\end{tabular}

process with Shima, she agreed and said, "You're right, and it hurts...." In the second phase of the observation series, Shima followed the CSM protocols. During the interview after the implementation of CSM, Shima, astonished to see the results, said, "Giving teachers constructive feedback has always been one of my major concerns. I think one of the biggest advantages of implementing CSM is that we can provide teachers with much more useful feedback, which may result in improving their performance in the future." "But the teachers received feedback in the ILI observation too," I replied. "Yes, but they got 'immediate feedback.' They were not allowed to reflect on their performance or comment on the supervisor's opinions and recommendations," she replied. Shima believed that, by applying CSM, teachers obtain valuable feedback because both teachers and supervisors have enough time to think about the class events and interactions. It seemed to her that CSM helps the supervisor have a collaborative relationship with the teacher in such a way from which both teachers and supervisors may benefit. "I think that's what we are looking for... A supervision process which helps the teachers improve their teaching skills in a pleasant atmosphere with the least tension between teachers and supervisors," she confirmed. Shima also believed that applying CSM can radically change the traditional views about the supervisory role in a positive way that leads not only to the teachers' excellence but also to supervisors' improvement in both the teaching and supervising areas.

\section{Discussion}

This study set out to determine the impacts of implementing the Clinical Supervision Model (CSM) on supervisors' teaching perspectives and qualifications. By applying the Transcendental Phenomenology approach, the present study scrutinized the lived experiences of two experienced supervisors before and after implementing CSM in the supervision process. The investigation aimed to answer the following research questions:

(1) What are the potential impacts of taking a supervisory role on observers' critical thinking about pedagogical issues?

(2) What are the effects of implementing CSM on supervisors' educational philosophy and teaching style in practice?

The first question in this study sought to determine the effects of taking a supervisory role on observers' teaching career. Both participants claimed that classroom observation is mutually beneficial. Nima believed that taking a supervisory role provided him with invaluable experience and 
gave him scope for creativity in teaching. The most interesting finding was that both interviewees with years of teaching experience underscored the fact that they were sometimes inspired by the artistic creativity of some novice teachers. During the first interview, Shima asserted that, in the process of observing classes, she usually gets brilliant ideas about how to teach a problematic part of a unit, lead a controversial discussion, and deal with difficult students who are reluctant to participate in classroom activities. Shima also criticized the summative nature of the ILI's observation system and expressed her dissatisfaction with the dictatorial attitudes of some ILI supervisors.

Throughout the first interview, Nima asserted that observing different teachers with various teaching techniques and qualifications has helped him reflect upon his own teaching in order to improve it. He supported the unannounced observations of classes and affirmed that he would let other teachers observe his own classes without prior notice, too. The current study's findings seem to be consistent with a growing body of literature indicating there is a link between observers' organizational role and their critical thinking about pedagogical issues. As an example, participation in peer observation was discovered to be an effective and strong instructional strategy for increasing self-efficacy in teaching; in a study by Mousavi [26], peer observation improves the self-efficacy of the observing teacher and the didactic skills of the observer. The results also accord with a study conducted by Motallebzadeh et al [27] in which it became clear that peer observation could significantly influence the professional development of EFL teachers by providing reflection and collaboration and learning new teaching skills and strategies. The findings are also in agreement with Ahmed et al.'s [28] study which concluded that taking a supervisory role has beneficial effects on the teaching community within their workplace.

Regarding the second research question, the participants acknowledged that CSM promotes reflective notions and provides both teachers and supervisors with constructive feedback, which may lead to improved teaching quality. They claimed that CSM encourages collaborative interaction with teachers and helps them reduce the level of stress. Consequently, they can have a prominent role in the process of observation and share their views freely with supervisors. This way, supervisors may also gain a broader perspective on teaching strategies and techniques. This finding is well matched with the ideas of Wiedmer [11] who believed that clinical supervision provides an opportunity for collegial support, allowing for the exchange of ideas, andreflection on professional practice, as well as offering encouragement while working towards educational objectives. The present finding also corroborates with the ideas of Gorsy et al. [29] who reported that a model like the CSM could fill the gaps and overcome the problems of current teaching practice due to its organized structure that provides systematic feedback to the student teacher. Its structure allows both the supervisor and the collaborating faculty to reflect on their performance to further improve the teaching practice. There are some similarities between the attitudes expressed in this study and those described by Bulunz et al. [30]. They supported the idea that CSM is a highly structured and organized model of reflection and feedback which maximizes the effectiveness of classroom practice. It incorporates the skills and resources of all those involved in a collegiate environment.

It is worth noting that both participants held identical views about the powerful impact of applying CSM on defusing the traditional tension between supervisors and teachers. According to the views, implementing CSM can help supervisors create a convivial atmosphere in the postobservation meetings, which leads to supervisors' more effective documentation of the supervision process. The supervisors believed that CSM is intended to create a constructive relationship between teachers and supervisors and improve the educational achievements of the process on both sides. These results accord with the findings of Ibara [20], in which he contends that the use of CSM implies that the supervisor and the teacher actively participate in the decisions made and strive to develop a relationship throughout the supervision cycle in order to promote the long-term professional improvement of both teachers and supervisors.

One of the most significant findings to emerge from this study is that the collaborative nature of CSM protocols creates a window of opportunity for supervisors to improve their own teaching profession. This finding accords with a great deal of the previous works in the area of CSM including Peake, Nussbaum, and Tindell [4] as well as Worthen and McNeill [5], as cited in the work of Nel and Fouche [3], in which the authors contend that experiencing the features of CSM results in teachers and supervisor's professional growth.

\section{Conclusion}

Taken together, the results of the study suggest that taking on a supervisory role may have a dramatic effect on supervisors' teaching careers. The face-to-face interactions with a variety of teachers with different teaching strategies and techniques offer them a wonderful chance to refine their own teaching philosophy and perspectives. The second major result that can be drawn from the study findings is that the implementation of CSM could affect the supervisors' educational attitudes and perspectives in practice, too. Due to the collaborative nature of CSM, the traditional tension of teachers and supervisors would be defused. As a result, teachers would be allowed to share their views with supervisors freely in the postobservation meetings, which could be regarded as a valuable source of knowledge and experience.

4.1. Limitations and Suggestions for Further Research. The findings in this study are subject to the following limitations. The most important limitation which may affect the generalizability of the findings lies in the fact that, due to the lengthy process of data collection, the researcher noticed a certain reluctance among a large number of teachers and supervisors to participate in the project. On the other hand, considering the nature of the applied methodology, it was 
decided to focus on personal views and lived experience of only two supervisors. A further study could assess the longterm effects of implementing CSM on the qualifications of different teachers and supervisors with an infinite variety of views. The second limitation was the narrow scope and context of the study. The bureaucratic process of gaining the consent of different language institutes inevitably limited the scope of the study and complicated the process of data collection. Further studies could be conducted in different educational contexts with various teaching methods and objectives.

4.2. Pedagogical Implications. The following implications can be drawn from the present study: The results of the study indicate that there is a direct relationship between implementing CSM and the effective collaboration of teachers and supervisors which may be conducive to students' successful learning. It can inspire educational policy-makers to provide a supportive environment for teaching staff to apply the principles of CSM in order to improve the quality of teaching in the educational contexts.

Another important practical implication that can be drawn from the results of the present study is that achieving the desired results in the supervision process greatly depends on the "consultative" rather than dominant role of supervisors. As a matter of fact, by providing a stimulating environment, CSM redefines the traditional roles of teachers and supervisors and helps them arrive at mutual understanding on the principle purpose of classroom observation. The obvious implication is that teachers and supervisors should adopt a more collaborative and flexible approach to classroom supervision in order to achieve a satisfactory outcome in the process of CSM implementation.

\section{Appendix}

\section{A. Preinterview Session}

\section{A.1. Supervisor's Personal Information}

Pseudonym: .................

Age: .................

Years of experience: ............

Date:

\section{A.2. Interview Checklist}

(1) Document a short background information about the Participant.

(2) Discuss the main purpose of the study.

(3) Explain the procedure of Clinical Supervision Model (CSM) in great detail.

(4) Elaborate on data collection procedure (classroom observations and semistructure interviews).

(5) Ensure the privacy and anonymity of the participants and gain written consent.

\section{B. Questions for Semistructured Interview (Phase 1, before Implementing CSM)}

(1) How would you describe your job as a supervisor of the Iran Language Institute? How do you feel about your current position as a supervisor?

(2) Has your supervisory role affected your performance in your teaching career so far? If so, how? Please elaborate on your answer as much as possible.

(3) If a teacher's performance doesn't meet the requirements and standards of the course, how will you run the postobservation meeting?

(4) In what ways do you provide the teachers with negative feedback when you want to convey your dissatisfaction in the postobservation meetings?

(5) Have you ever learned anything new during your observations which made you change the way you teach the same content in your own classes?

(6) How does the ILI's current observation system help the teachers improve their teaching quality? How can it be more effective?

(7) If the teachers want to observe your own classes unexpectedly, without prior notice as you always do, will you allow them to do so?

(8) In what ways do you usually support preservice and novice teachers?

(9) Do you have any interactions with other supervisors regarding classroom observations and teachers' performance?

(10) What changes would you apply in the current observation system if you were a member of the ILI Planning and Research Department?

\section{Questions for Semistructured Interviews (Phase 2, after Implementing CSM)}

(1) Did you notice any significant changes in the teacher's attitude and performance when you applied the protocols of CSM?

(2) Did you experience any specific challenges during the implementation of CSM? If so, what do you think should be done to resolve it?

(3) Some scholars believe that implementing CSM helps the teachers reduce the level of stress during the observation and make them more efficient as a consequence. Can it be true on the basis of your experience?

(4) Can the implementation of CSM influence supervisors' general notions and attitudes towards their supervisory role and teaching career? If so, how exactly? Please elaborate on your answer as much as possible.

(5) According to the protocols of CSM, teachers need to be aware of supervisors' presence in advance. To what extent do you agree with this notion? 
(6) Did you notice any significant differences in the handling of postobservation meetings between CSM and other methods of supervision?

(7) If you were a preservice or novice teacher, how would you prefer to be observed? Is there any special privilege on the side of the teachers in the process of CSM?

(8) Can the implementation of CSM affect the supervisors' teaching philosophy in practice? If so, how?

(9) If you were allowed to choose your own supervision strategy, would you apply CSM to your career?

(10) In your opinion, what are the advantages and disadvantages of CSM?

\section{Data Availability}

The data used to support the findings of this study are included within the article.

\section{Disclosure}

The authors confirm that the ideas expressed in the submitted article are of their own and not those of any official position of the institution or funder.

\section{Conflicts of Interest}

The authors declare that they have no conflicts of interest.

\section{References}

[1] K. A. Acheson and M. D. Gall, Techniques in the Clinical Supervision of Teachers: Preservice and Inservice Applications, Longman Publishers, White Plains, NY, USA, 4th edition, 1997.

[2] M. O'Leary, Classroom Observation: A Guide to the Effective Observation of Teaching and Learning, Routledge, England, UK, 2020.

[3] L. Nel and P. Fouche, "Experiences of master's students regarding clinical supervision in an applied psychology programme in South Africa," Teaching in Higher Education, vol. 22, no. 1, pp. 30-43, 2017.

[4] T. H. Peake, B. D. Nussbaum, and S. D. Tindell, "Clinical and counseling supervision references: trends and needs," Psychotherapy: Theory, Research, Practice, Training, vol. 39, no. 1, pp. 114-125, 2002.

[5] V. Worthen and B. W. McNeill, "A phenomenological investigation of "good" supervision events," Journal of Counseling Psychology, vol. 43, no. 1, pp. 25-34, 1996.

[6] M. L. Cogan, Clinical Supervision, Houghton Mifflin, Boston, MA, USA, 1973.

[7] R. Goldhammer, "Clinical supervision: special methods for the supervision of teachers," Holt McDougal, 1969.

[8] P. P. Grimmett, "Clinical supervision and teacher thought processes," Canadian Journal of Education, vol. 6, no. 4, pp. 23-39, 1981.

[9] R. J. Marzano, T. Frontier, and D. Livingston, Effective Supervision: Supporting the Art and Science of Teaching, ASCD, 2011.
[10] R. F. Bruce and L. Hoehn, "Supervisory practice in Georgia and Ohio," Annual Meeting of the Council of Professors of Instructional Supervision, 1990.

[11] T. L. Wiedmer, "Instructional improvement: the goal of clinical supervision," The Teacher Educator, vol. 30, no. 3, pp. 6-18, 1995.

[12] A. T. Bello and J. H. Olaer, "The influence of clinical supervision on the instructional competence of secondary school teachers," Asian Journal of Education and Social Studies, vol. 12, no. 3, pp. 42-50, 2020.

[13] A. Rahmat, R. Badu, and R. Djibu, "Implementation of clinical supervision to improve teacher performance in managing learning in tk Dungaliyo gorontalo District," International Journal of Innovations in Engineering Research and Technology, vol. 7, no. 9, pp. 67-74, 2020.

[14] S. Babaeer, A Transcendental Phenomenological Study of Supervision in Teacher Preparation in Saudi Arabia, Doctoral Dissertation, University of South Florida, Tampa, FL, USA, 2021.

[15] L. Baecher and R. W. Burns, "Advancing supervision in clinically-based teacher education," Journal of Educational Supervision, vol. 3, no. 1, pp. 1-4, 2020.

[16] H. Marashi and M. Bani-Ardalani, "Using clinical supervision to lower EFL teachers' burnout," Issues in Language Teaching, vol. 6, no. 2, pp. 261-289, 2017.

[17] M. McGhee and M. Stark, "Promoting collegial teacher supervision: applying solution-focused strategies in a clinical supervision cycle," International Journal of Leadership in Education, vol. 21, no. 6, pp. 726-740, 2018.

[18] A. Bencherab and A. Al Maskari, "Clinical supervision: a genius tool for teachers' professional growth," The Universal Academic Research Journal, vol. 3, no. 2, pp. 51-57, 2020.

[19] W. S. Hopkins and K. D. Moore, "Clinical supervision: a neoprogressive approach," The Teacher Educator, vol. 30, no. 3, pp. 31-43, 1995.

[20] E. C. Ibara, "Exploring clinical supervision as instrument for effective teacher supervision," Africa Education Review, vol. 10, no. 2, pp. 238-252, 2013.

[21] L. Cohen, L. Manion, and K. Morrison, "Research methods in education," Research Methods in Education, vol. 7, 2011.

[22] J. W. Creswell and C. N. Poth, Qualitative Inquiry and Research Design: Choosing Among Five Approaches, SAGE Publications, Thousand Oaks, CA, USA, 2016.

[23] C. Moustakas, Phenomenological Research Methods, SAGE Publications, Thousand Oaks, CA, USA, 1994.

[24] S. M. Laverty, "Hermeneutic phenomenology and phenomenology: a comparison of historical and methodological considerations," International Journal of Qualitative Methods, vol. 2, no. 3, pp. 21-35, 2003.

[25] E. Husserl, "Psychological and Transcendental Phenomenology and the Confrontation with Heidegger (1927-1931): The Encyclopaedia Britannica Article, The Amsterdam Lectures," Phenomenology and Anthropology and Husserls Marginal Notes in Being and Time and Kant and the Problem of Metaphysics, vol. 6, 1997.

[26] S. M. Mousavi, "The effect of peer observation on Iranian EFL teachers' self-efficacy," Procedia-Social and Behavioral Sciences, vol. 136, pp. 181-185, 2014.

[27] K. Motallebzadeh, M. Hosseinnia, and J. G. H. Domskey, "Peer observation: a key factor to improve Iranian EFL teachers' professional development," Cogent Education, vol. 4, no. 1, Article ID 1277456, 2017. 
[28] E. Ahmed, Z. S. Nordin, S. R. Shah, and M. A. Channa, "Peer observation: a professional learning tool for English language teachers in an EFL institute," World Journal of Education, vol. 8, no. 2, pp. 73-87, 2018.

[29] E. Gürsoy, N. Bulunuz, Ş. Baltac1-Göktalay, M. Bulunuz, J. Kesner, and U. Salihoğlu, "Clinical supervision model to improve supervisory skills of cooperating teachers and university supervisors during teaching practice," HU Journal of Education, vol. 1, pp. 191-203, 2013.

[30] N. Bulunz, E. Gursoy, J. Kesner, S. Baltaci Goktalay, and U. M. Salihoglu, "The implementation and evaluation of a clinical supervision model in teacher education in Turkey: is it an effective method," Educational Sciences: Theory and Practice, vol. 14, no. 5, pp. 1823-1833, 2014. 